\title{
Successful closure of pulmonary artery aneurysm in a patient with Hughes-Stovin syndrome
}

\author{
Shokoufeh Hajsadeghi', Roozbeh Naghshin', Mehrdad Nikfam', Ali Pourmojarab', \\ Sayfollah Abdi ${ }^{2}$ \\ 'Rasoul Akram Medical Center, Iran University of Medical Sciences, Iran \\ ${ }^{2}$ Rajaie Cardiovascular, Medical \& Research Center, Iran University of Medical Sciences, Iran
}

\begin{abstract}
Hughes-Stovin syndrome is a syndrome of unknown etiology characterized by thrombophlebitis and pulmonary aneurysms. Less than 40 cases have ever been reported in the English medical literature. We are reporting a 38-year-old man with a history of right ventricular thrombus and also deep vein thrombosis who presented with massive hemoptysis.

Initial work-up revealed a round opacity on chest roentgenogram which later was diagnosed as a pulmonary artery aneurysm on subsequent imaging studies. Based on the history of thrombophlebitis with this newly diagnosed pulmonary artery aneurysm a diagnosis of Hughes-Stovin syndrome was made. The patient was treated with endovascular coiling of the aneurysm. A sixteen-month follow up was uneventful. To our knowledge, this is the first case of HSS ever reported from Iran and the fourth to have a cardiac chambers involvement in the course of the disease.
\end{abstract}

Key words: pulmonary artery, aneurysms, Hughes-Stovin syndrome

Acta Angiol 2017; 23, I: 20-24

\section{Introduction}

First described by Drs. John Patterson Hughes and Peter George Ingle Stovin in 1959, Hughes-Stovin syndrome (HSS) is a rare clinical entity characterized by thrombophlebitis and pulmonary aneurysms $[\mathrm{I}, 2]$. It is a syndrome of unknown etiology that usually affects young men in their second to third decade of life presenting with symptoms such as cough, dyspnea, hemoptysis, fever and chest pain. The expected archetype of the clinical course can be categorized into three different phases [3, 4]: a) symptoms of thrombophlebitis, b) formation of large pulmonary and/or bronchial aneurysms and c) aneurysmal rupture leading to massive hemoptysis which is the leading cause of mortality in patients with HSS. We herein are reporting a 38-year-old man with HSS.

\section{Case study}

This is a 38-year-old Iranian male patient seen at the Pulmonary Diseases Department of our hospital in February 2015 with a 40 day history of repeated episodes of hemoptysis each about $200 \mathrm{~mL}$ with exertional dyspnea, cough, nocturnal fever (not responsive to antipyretic medications) and pleuretic chest pain. Earlier in 2010, he had been worked up in this hospital for acute swelling of left lower limb and upon diagnosis of acute deep vein thrombosis (DVT), was put on a course of anticoagulation (warfarin). 14 days after the initial treatment, acute hemorrhage into the thyroid gland occurred and he underwent partial thyroidectomy. Since then, he was on levothyroxine and low-dose warfarin for about one year with regular follow-ups, maintaining INR in therapeutic range. For the first time in 2012, he had had episodes of hemoptysis with cough, 


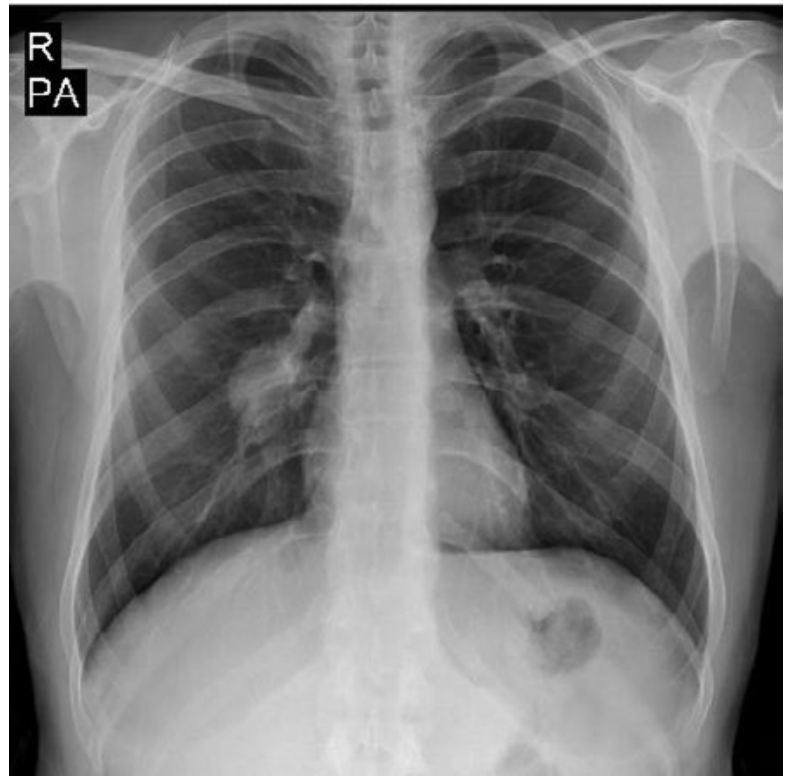

Figure I. Standard posteroanterior chest X-ray showing a round opacity in the right lower lobe

hemosputum and dyspnea. All initial laboratory tests had been within the normal limits. Transthoracic echocardiogram (TTE) revealed an echogenic mass lesion measuring about $36 * 20 \mathrm{~mm}$ in the right ventricle (RV) attached to the interventricular septum adjacent to the inferior vena cava (IVC). Subsequent dynamic cardiac MRI with gadolinium stipulated the mass as an RV clot (thrombosis). Fibroptic bronchoscopy and computerized tomographic angiography of the pulmonary arteries (CTA) were unremarkable. Further laboratory tests including coagulation panel, anti-cardiolipin and anti-phospholipid antibodies and tests for human immunodeficiency virus $I$ and 2 , hepatitis B surface antigen were normally excepted for Factor $V$ Leiden. The patient was commenced on intravenous unfractionated heparin followed by $5 \mathrm{mgs}$ of warfarin daily. A follow-up echocardiography two months later revealed no thrombosis. On the current admission, the patient was in a good general condition, with normal vital signs. Physical examination was unremarkable. There were no signs of uveitis, oral or genital ulcerations or scars of healed genital ulcers. Primary blood tests unveiled mild microcytic anemia and a slightly elevated CRP $(12 \mathrm{mg} / \mathrm{L})$. Coagulation studies were normal (the patient discontinued warfarin without being told 30 days prior to the admission). Plain chest roentgenogram divulged a round, well-defined opacity in the right lower lobe without air bronchograms (Fig. I). A consecutive contrast-enhanced computed tomography (CECT) of thorax affirmed a single fusiform type of aneurysmal dilatation of the descending branch of the right pulmonary artery (Figs. 2, 3). Further laboratory studies, namely, antinuclear antibodies (ANA), anti-dsDNA antibodies, anti-neutrophil cytoplasmic antibodies (cANCA,

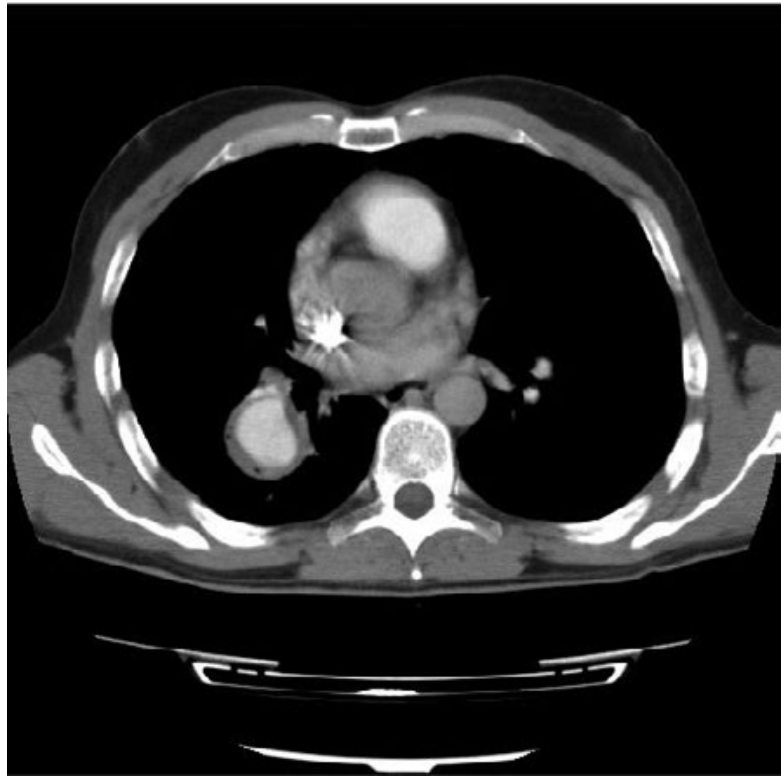

Figure 2. Contrast-enhanced computed tomography (CECT) of the chest, axial view showing aneurysmal dilatation of the descending branch of the right pulmonary artery with intramural thrombus formation
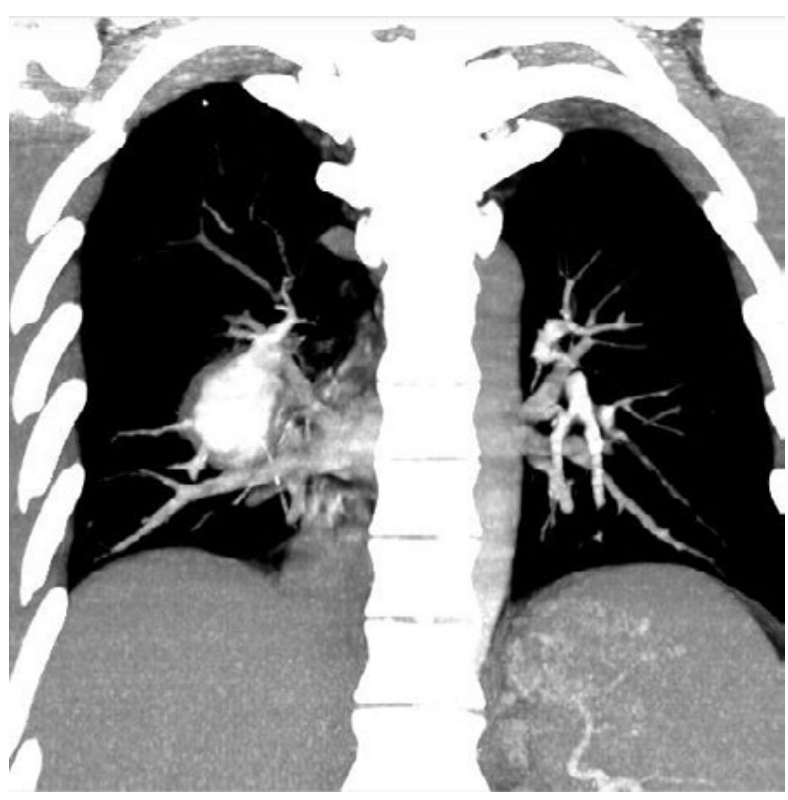

Figure 3. Coronal multiplanar reformat (MPR) image showing the aneurysm with a round opacity in the right lower lobe

pANCA), complement levels and anticardiolipin antibodies were all within the acceptable range. With this newly detected pulmonary artery aneurysm (PAA), an indisputable history of thrombophlebitis including both the deep veins and cardiac chambers, absence of typical signs of Behçet's disease (BD) in a man of this age, a diagnosis of Hughes-Stovin syndrome was made.

In contemplation of the treatment options based on the literature, regarding massive hemoptysis and the 
singular PAA, an interventional approach was preferred because of lower procedural risk and higher chance to get effective access to bleeding site with guidance of preprocedural CT angiography.

After insertion of 7F short sheath at both right and left femoral veins, two diagnostic catheters including pigtail and right Judkins were first introduced to pulmonary artery (Fig. 4) then to right main and lower segmental arteries for precise injection and delineating the anatomy of aneurysm especially the inflow neck.

For exclusion of the aneurysm, there were some alternatives including coil, vascular plug, stent graft

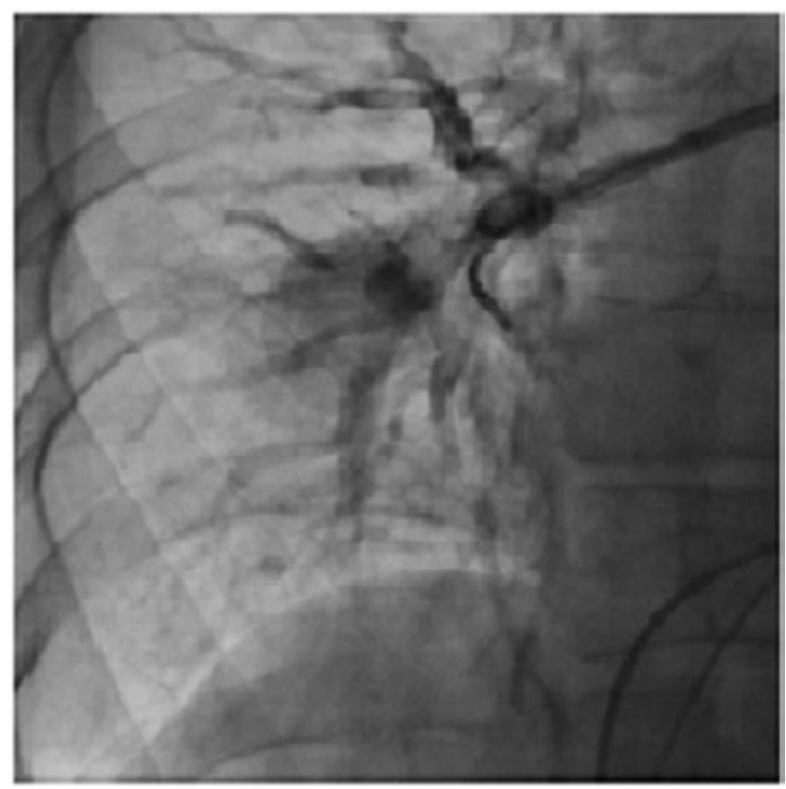

Figure 4. A pigtail and a right Judkins catheter are introduced to the pulmonary artery catheter embolization, etc., but we closed the aneurysm easily and effectively with a muscular VSD closure device, size number 14, using a long $10 \mathrm{~F}$ sheath (Fig. 5). The thrombosis of aneurysm sac was shown in the angiography room by contrast injection using the pigtail catheter (Fig. 6). The next day after the procedure, CT angiography was repeated which revealed near complete thrombosis in the aneurysm and also extension into the segmental branches which induced oligemia in the right lower lobe.

A sixteen-month follow-up was uneventful and the patient is doing well now. To our knowledge, this is the first HSS patient ever reported from Iran and the fourth case to have a cardiac chambers involvement during the course of the disease.

\section{Discussion}

Hughes-Stovin syndrome was named after two British physicians, John Patterson Hughes and Peter George Ingle Stovin who first described the characteristics of a syndrome (deep venous thrombosis and pulmonary artery aneurysms) in a total of four male patients in 1959 [I]. Since then, less than forty cases of HSS have been reported in the English medical literature [2]. This syndrome predominantly affects men in their second and third decades of life. The reported cases ranged between 12 to 48 years of age $[5,6]$ but Korunic et al. reported a 70-year-old male patient with HSS [7]. Only seven women have been reported presenting this syndrome thus far [8]. The exact etiology and pathogenesis of HSS remains widely unclear. Several proposed theories have attempted to explain the manifestations of this entity $[9,10]$. These encompass

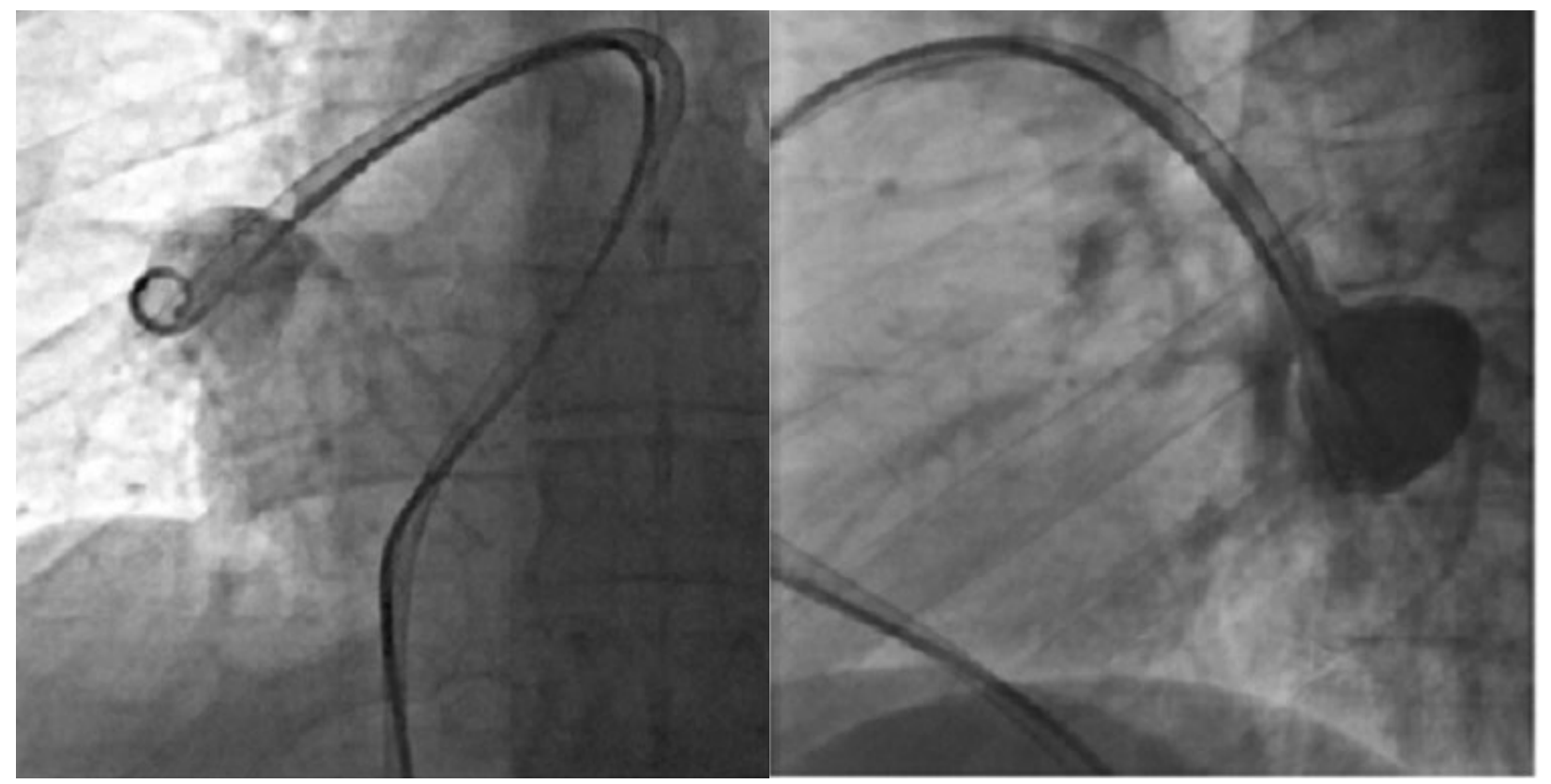

Figure 5. The pulmonary artery aneurysm is closed with a muscular VSD closure device 


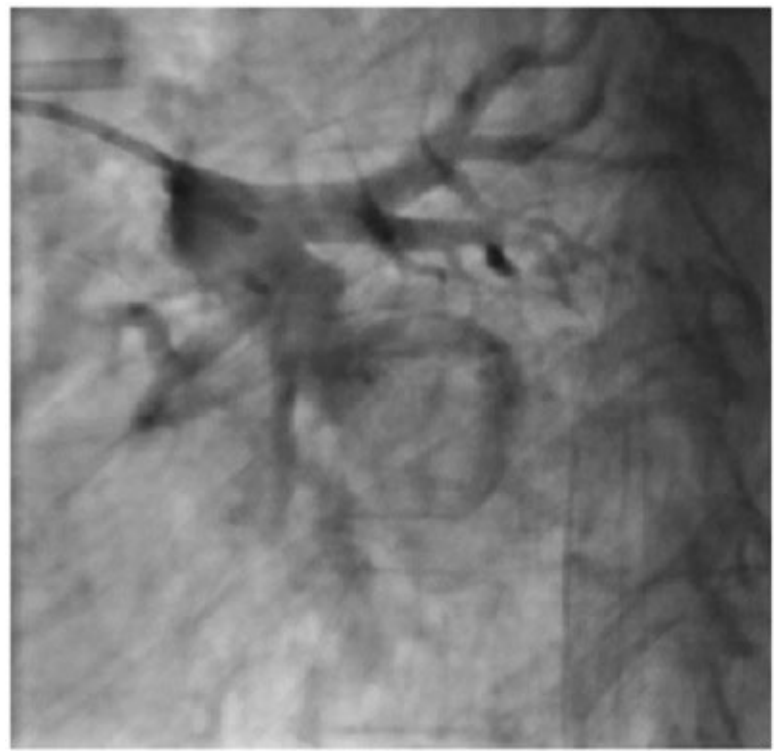

Figure 6. The thrombosis of aneurysmal sac is shown after contrast

infectious causes and angiodysplaisa. Another debatable hypothesis accounts for the vascular changes as the primary cause which is much closer to the original idea proposed by Hughes and Stovin who believed that the structural changes in the bronchial arteries impaired the provision of adequate nutrition to the pulmonary arteries through the vasa vasorum. In turn, these events led to inflammation, damage to the elastic tissue and creation of arterial aneurysms [I]. Actually, weakening of the arterial wall from the subsequent inflammation is the likely mechanism [I I]. The clinical course of HSS can be categorized into three different phases [3, 4]: a) symptoms of thrombophlebitis, b) formation of large pulmonary and/or bronchial aneurysms and c) aneurysmal rupture leading to massive hemoptysis Patients can have seizures, diplopia and cephalalgia secondary to raised intracranial pressure consequent to cerebral venous sinus thrombosis [1, 12] akin to the patient reported by Chalazonitis et al. [13]. About 25\% of patients with HSS develop thromboembolism, arterial aneurysms and vascular occlusions. The distribution of the vascular component of the syndrome is as follows: arterial (7\%), venous (25\%) or both (68\%) [14]. Some authors have proposed that HSS may actually be a partially manifested Behçet's disease (BD) based on their similar findings $[12,15,16]$. In fact, HSS and BD are the only vasculitides associated with the development of pulmonary artery aneurysms yet most HSS patients do not have typical manifestations of BD such as oral and genital ulcers, uveitis or arthralgia. The laboratory findings in HSS patients are non-specific. The patients can have leukocytosis, anemia, raised ESR and elevated CRP. As noted earlier, anemia and elevated CRP were present in the initial assessment of our patient. Chest radiography may reveal round opacities and hilar enlargement. Helical computed tomography (HCT) and magnetic resonance imaging have been reported as non-invasive method useful for the diagnosis of PAA [8]. Traditionally, conventional angiography has been regarded as a gold standard for the diagnosis of pulmonary artery aneurysms [2]. It also aids in assessment of angiodysplastic bronchial arteries in HSS. The characteristic picture seen is aneurysmal formation proximal to the occluded segments while distal to the interruption, signs of hypoperfusion are observed [17]. However, it should be noted that selective pulmonary angiography can be hazardous as it carries the risk of aneurysm rupture [ 18]. Today, in fact, multi-detector row helical CT angiography offers more precise visualization of large systemic arteries than does conventional angiography [2], and is considered the diagnostic method of choice in most of the patients. Classic histopathologic findings of HSS [ I, 2, I 2] include diffuse dilatation and partial occlusion of the aneurysmal arteries, perivascular infiltration that is predominantly lymphomonocytic and diffuse proliferative sclerosis. In the pulmonary veins, fibrosis and muscular medial thickening is seen. Due to the lack of controlled trials, there are no standard treatment guidelines for HSS and various treatment modalities have been described in the management of this disease [2]. Most commonly, immunosuppressive therapy involving a combination regimen of glucocorticoids and cyclophosphamide has been employed as a first line medical management in the treatment of HSS, although its effectiveness remains to be fully established [19]. Gortani et al. reported successful treatment of refractory HSS with infliximab in a 12-year-old Albanian boy [20]. Anticoagulation is generally contraindicated due to an increased risk of fatal hemorrhage. However, it may be employed with great vigilance in a few carefully evaluated circumstances where the benefits are believed to significantly outweigh the risks [2]. We decided not to restart anticoagulation therapy owing to the massive hemoptysis. For the cases of massive hemoptysis due to large pulmonary aneurysms or those with lesions confined to one segment or one lung (as of our case), surgical removal of the aneurysm, interventional closure, lobectomy or pneumonectomy can be carried out based on the data from published case reports [2]. Durieux et al. [2I] used surgical intervention for three cases of HSS who had isolated pulmonary aneurysms and none of them showed any signs of recurrence on subsequent follow-ups.

\section{Conclusions}

Hughes-Stovin syndrome is a very rare clinical entity. Hence the exact etiology is unknown and no widely accepted diagnostic criteria have been suggested, the 
diagnosis requires a high level of clinical suspicion. Since early diagnosis and treatment is the key for improving the outcome of HSS patients, having the syndrome in mind as a differential diagnosis for PAA, it is imperative to consider HSS in the differential diagnosis.

\section{References}

I. Huhhes JP, Stovin PG. Segmental pulmonary artery aneurysms with peripheral venous thrombosis. $\mathrm{Br} J \mathrm{Dis}$ Chest. 1959; 53(I): 19-27, indexed in Pubmed: 13618502.

2. Khalid U, Saleem T. Hughes-Stovin syndrome. Orphanet J Rare Dis. 201 I; 6: 15, doi: 10.1186/1750-1172-6-15, indexed in Pubmed: $\underline{21489283}$.

3. Reimold WV, Emmrich ], Harmjanz D, et al. [Multiple aneurysms of the pulmonary artery following recurrent septic pulmonary embolism (Hughes-Stovin syndrome). Report of I case]. Arch Klin Med. 1968; 215(I): I-I8, indexed in Pubmed: 5756378.

4. Khali A, Parrot A, Fartoukh M, et al. Images in cardiovascular medicine. Large pulmonary artery aneurysm rupture in Hughes-Stovin syndrome: multidetector computed tomography pattern and endovascular treatment. Circulation. 2006; II4(10): e380e38I, doi: $10.1161 /$ CIRCULATIONAHA.106.614636, indexed in Pubmed: 16952990.

5. Grembiale DR, Calderazzo M, Pelaia G, et al. A case of HughesStovin syndrome associated with Budd-Chiari syndrome. Case Rep Clin Pract Rev. 2002; 3: 92-94.

6. Curci N, Bilfinger T. Successful multimodality treatment of a young woman with HughesStovin syndrome. Am Surg. 2012; 78(5): 304-305.

7. Huzjan Korunic R, Vukelic Markovic M, Tekavec Trkanjec J, et al. Hughes-Stovin Syndrome: a case report of man with hemoptysis due to pulmonary and bronchial arterial aneurysms. ESTI. 2014: P-0019, doi: 10.1594/esti2014/P-0019.

8. El Aoud S, Frikha F, Snoussi M, et al. Moderate hemoptysis caused by hughes-stovin syndrome. Clin Pract. 20 14; 4(3): 647 , doi: $10.4081 /$ cp.2014.647, indexed in Pubmed: 25568765.

9. Ammann ME, Karnel F, Olbert F, et al. Radiologic findings in the diagnosis of Hughes-Stovin syndrome. AJR Am J Roentgenol. 1991; 157(6): 1353-1354, doi: 10.2214/ajr.157.6.1950888, indexed in Pubmed: 1950888.
10. Roberts DH, Jimenez JF, Golladay ES. Multiple pulmonary artery aneurysms and peripheral venous thromboses - the Hughes Stovin syndrome. Report of a case in a 12-year-old boy and a review of the literature. Pediatr Radiol. 1982; 12(4): 214-216, indexed in Pubmed: $\underline{6752855}$.

II. Charlton RW, Du Plessis LA. Multiple pulmonary artery aneurysms. Thorax. 1961; 16: 364-37I, indexed in Pubmed: 13878292.

12. Choh NA, Jehangir M, Mir KM, et al. Hughes-Stovin syndrome: A rare cause of hemoptysis. Lung India. 20I I; 28(4): 285-286, doi: $10.4103 / 0970-2113.85691$, indexed in Pubmed: 22084543.

13. KOPP W. Pulmonary Artery Aneurysms with Recurrent Thrombophlebitis. Annals of Internal Medicine. 1962; 56(I): 105, doi: 10.7326/0003-4819-56-I-105.

14. François MF. Is Hughes-Stovin syndrome a particular expression of Behçet's disease? Chest. 1983; 83(2): 288, indexed in Pubmed: 6217953.

15. Chalazonitis AN, Lachanis SB, Mitseas P, et al. Hughes-Stovin syndrome: a case report and review of the literature. Cases J. 2009; 2: 98, doi: 10.1186/1757-1626-2-98, indexed in Pubmed: $\underline{19178695}$.

16. Al-jahdali H. Massive hemoptysis and deep venous thrombosis presenting in a woman with Hughes-Stovin syndrome: a case report. J Med Case Rep. 2010; 4: 109, doi: 10.1186/1752-19474-109, indexed in Pubmed: 20409300.

17. Mahlo HR, Elsner K, Rieber A, et al. New approach in the diagnosis of and therapy for Hughes-Stovin syndrome. AJR Am J Roentgenol. 1996; 167(3): 817-818, doi: 10.2214/ajr.167.3.8751710, indexed in Pubmed: 8751710.

18. Weintraub JL, DeMayo R, Haskal ZJ, et al. SCVIR annual meeting film panel session: diagnosis and discussion of case I: HughesStovin syndrome. J Vasc Interv Radiol. 200I; 12(4): 53 I-534, indexed in Pubmed: 11287545.

19. Erkan F. Rare diseases bullet 12: Pulmonary manifestations of Behcet's disease. Thorax. 2001; 56(7): 572-578, doi: 10.1136/ thorax.56.7.572.

20. Gortani G, Starc M, Tubaro M. Successful treatment of refractory hughes stovin syndrome with infliximab. Pediatric Rheumatology. 2014; I2(Suppl I): P352, doi: 10.1 |86/I546-0096-|2-s|-p352.

21. Durieux P, Bletry $O$, Huchon G, et al. Multiple pulmonary arterial aneurysms in Behcet's disease and Hughes-Stovin syndrome. Am J Med. 198I; 7I(4): 736-74I, indexed in Pubmed: 7282759 . 